Now, integrating the equation (7) between the limits 0 and $u$, we have

$$
\lambda-p=\int_{0}^{u}\left\{u p(1+u p)^{2}+\left(2 p^{2}+3 u p^{3}\right)\right\} d u .
$$

Suppose that $0<\lambda \leq M$. Then we have proved that, for any $\lambda, u p \rightarrow 0$ as $u \rightarrow \infty$, and that $p(u, \lambda)<p(u, M)$. Hence $p=o(1 / u), p^{2}=\mathrm{o}\left(1 / u^{2}\right), u p^{3}=\mathrm{o}\left(1 / u^{3}\right)$ : and therefore $\int_{0}^{u}\left(2 p^{2}+3 u p^{3}\right) d u$ is uniformly convergent as $u \rightarrow \infty$. But since $p \rightarrow 0$ and $p(u, \lambda)$ $<p(u, M)$ as $u \rightarrow \infty$, the left hand side of (16) is uniformly convergent to $\lambda$. Therefore, since for all large enough $u,(1+u p)>\frac{1}{2}$, it follows at once that $\int_{0}^{u} u p d u$ is uniformly convergent when $u \rightarrow \infty$ : and the same is therefore true of $\int_{0}^{\infty} p d u$.

(v) We may write (16) in the form

$$
\lambda-p=-\int_{u}^{0}\left\{u p(1+u p)^{2}+\left(2 p^{2}+3 u p^{3}\right)\right\} d u .
$$

We have proved that for all small enough $\lambda$, say $\lambda \leq H$, up $\rightarrow 0$ as $u \rightarrow-\infty$. In this event, it follows, by precisely the reasoning of (iv) above, that $\int_{-\infty}^{0} u p d u$ and $\int_{-\infty}^{0} p d u$ are uniformly convergent.

\title{
AN ELEMENTARY SOLUTION OF TWO STRESS CONCENTRATION PROBLEMS IN THE NEIGHBOURHOOD OF A HOLE*
}

\author{
Br W. T. KOITER (Technische Hogeschool, Delft)
}

1. Introduction. The problem of the stress concentration around a central circular hole in a strip in tension (or in bending in its plane) has been investigated extensively. The theoretical solution of this problem has been given by Howland [1]. However, Howland's numerical calculations were restricted to hole diameters which do not exceed half the strip width, and his method requires a rapidly increasing numerical effort for larger holes. Experimental investigations have yielded valuable data on the stress concentration factor for large holes. On the basis of these experimental results Heywood [2] conjectures that the stress concentration factor, referred to the nominal stress in the net section, tends to the value 2 if the hole diameter approaches to the strip width. This conjecture is confirmed by the elementary analysis in Sec. 2 .

Recently the more complicated problem of a central spherical hole in a cylindrical bar in tension has been investigated by Ling [3]. His numerical cvaluation has also been restricted to a hole radius equal to $1 / 4$ and $1 / 2$ times the bar radius. Here again the numerical effort, required in evaluation of the theory, increases rapidly with increasing hole radius. According to Ling "the value 1 for the stress concentration factor $K$ (referred to the nominal stress in the net section) in the limiting case $\lambda=1$ (where $\lambda$ is the ratio of hole radius to bar radius) can be visualized readily from physical considerations of the cylinder" [3, p. 391], and the graph of his results [3, Fig. 2] has been completed for the entire range $0<\lambda<1$. However, Ling's argument, which has been cited above, is incorrect, as will be shown in Sec. 3. The correct limiting value of the stress concentration

*Received May 18, 1956. 
factor is

$$
\lim _{\lambda \rightarrow 1} K=\frac{(6-4 \nu)(1+\nu)}{5-4 \nu^{2}},
$$

where $\nu$ is Poisson's ratio. Ling's graph for the stress concentration factor $K$ as a function of $\lambda$ should be corrected accordingly.

Our elementary solutions for the stress concentration problems for the strip with a large circular hole and the cylindrical bar with a large spherical hole are based on beam theory and shell theory respectively. It might be objected that both these theories are approximate in character. On the other hand, it may be contended that the accuracy of beam and shell theories increases indefinitely when the "slenderness" of beam and shell increase indefinitely, and the limiting values for the stress concentration factor $K$ for $\lambda \rightarrow 1$ should therefore be correct.

Nevertheless, a more rigorous investigation is considered worthwhile, the more so because it may then be possible to investigate the manner in which the limiting value is approached. A suitable method is mentioned in Sec. 4 and some preliminary results for the strip problem, confirming the result of beam theory, are given.

2. Strip with a large circular hole. The strip with a large circular hole $(\delta \ll R)$ is pictured in Fig. 1, and the upper part to the right of the minimum section is given on a

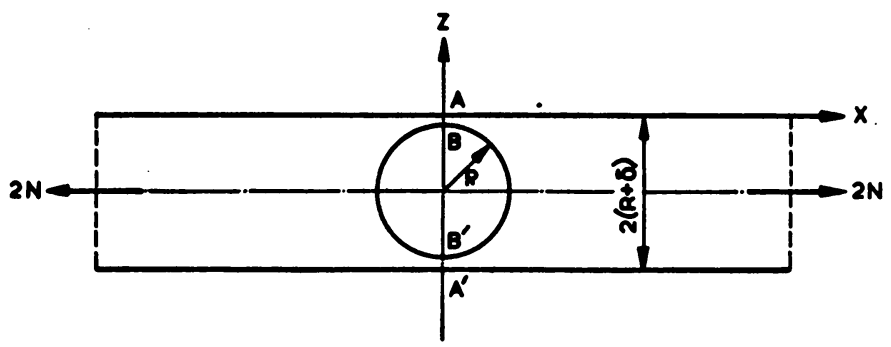

FIG. 1 ,

larger scale in Fig. 2. It is obvious from symmetry that no shear force is transmitted by the minimum cross-section $A B$. On the other hand, in order to maintain this crosssection in a vertical position, which is again obviously necessary on account of symmetry, a positive bending moment $M_{0}$ must occur in this cross-section.

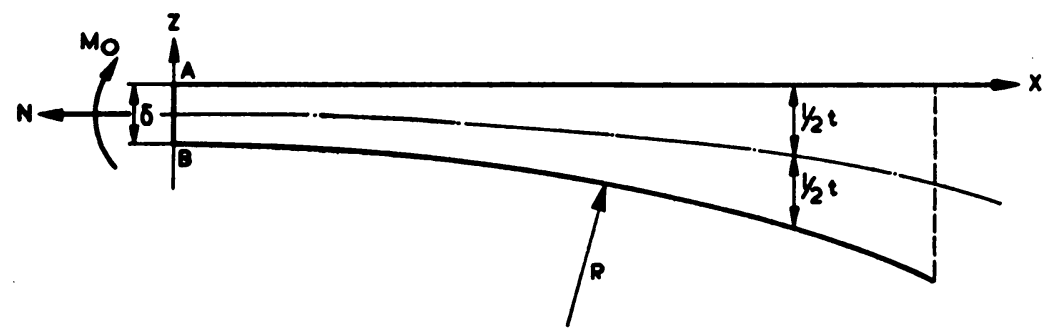

Frg. 2

An element of the "beam" in Fig. 2 has been drawn in Fig. 3. Obviously the shear force is zero and the normal force has the constant value $N$. 


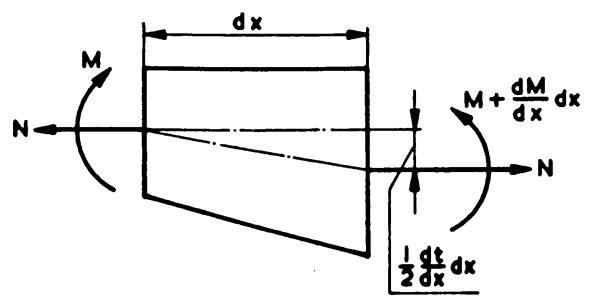

Fig. 3

Equilibrium of moments requires

$$
\frac{d M}{d x}=-\frac{1}{2} N \frac{d t}{d x} .
$$

In the neighbourhood of the minimum cross-section $x=0$ the height $t$ of the beam is approximated by

$$
t=\delta+\frac{x^{2}}{2 R} .
$$

Hence, Eq. (2) may be written in the form

$$
\frac{d M}{d x}=-\frac{1}{2} N \frac{x}{R} .
$$

Because $d t / d x$ is small in the neighbourhood of $x=0$, we may apply the beam formula

$$
M=E I \frac{d^{2} w}{d x^{2}}=\frac{1}{12} E h t^{3} \frac{d^{2} w}{d x^{2}},
$$

where $w$ is the deflection (positive upwards), $h$ is the strip thickness and $E$ is Young's modulus. Equation (4) now reads

$$
\frac{1}{12} E h \frac{d}{d x}\left(t^{3} \frac{d^{2} w}{d x^{2}}\right)=-\frac{1}{2} N \frac{x}{R} .
$$

The general solution of (6) contains three integration constants. Two boundary conditions are supplied by the requirement that $w$ and its derivatives tend to zero for $x \rightarrow \infty$ because the deformation is negligible for large values of $x / \delta$. The third boundary condition is that the slope $d w^{\prime} d x$ at the minimum cross-section $x=0$ is zero. Trying a power series solution in $t^{-1}$ it is immediately seen that

$$
w=-\frac{2 N R}{\operatorname{Eh}\left(\delta+x^{2} / 2 R\right)}
$$

satisfies both the differential equation and the boundary conditions.

The bending stress at the inner edge of the beam is given by

$$
\sigma_{b}=\frac{1}{2} E t \frac{d^{2} w}{d x^{2}} .
$$




\section{Putting}

$$
\frac{x}{(R \delta)^{1 / 2}}=\xi
$$

we obtain for the sum of bending stress and tensile stress (due to the tensile force $N$ ) at the inner edge and outer edge respectively

$$
\sigma_{i}=\frac{N}{h \delta} \frac{2-\xi^{2}}{\left(1+\xi^{2} / 2\right)^{2}}, \quad \sigma_{0}=\frac{N}{h \delta} \frac{2 \xi^{2}}{\left(1+\xi^{2} / 2\right)^{2}} .
$$

The maximum stress occurs at the inner edge at $x=0$; its value is $2 N / h \delta$, and the stress concentration factor $K=2$.

3. Cylindrical bar with a large spherical hole. Figure 1 also depicts a meridional section of a cylindrical bar with a large spherical hole $(\delta \ll R)$, whereas Fig. 2 may be regarded again as an enlarged picture of the upper part to the right of the minimum section. This part is now considered as a cylindrical shell of variable thickness $t$, given by (3). An element of this shell of circumferential angle $d \varphi$ is drawn in Fig. 4, where the bending moment $m$ and the tensile and shear forces $n$ and $q$ are defined per unit circumferential angle. Obviously the tensile force $n$ is constant. Equilibrium of moments

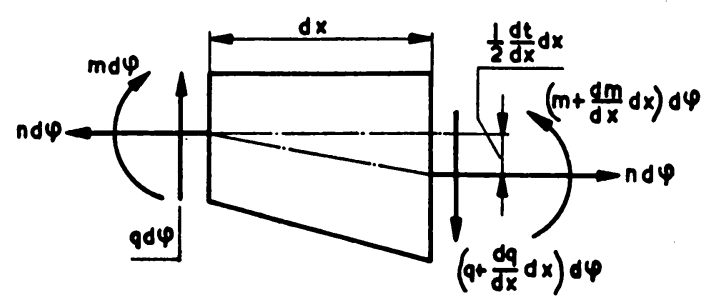

FIg. 4

requires

$$
\frac{d m}{d x}=q-\frac{1}{2} n \frac{d t}{d x},
$$

whereas the equilibrium of radial forces is expressed by

$$
\frac{d q}{d x}=-t \sigma_{\varphi}
$$

where $\sigma_{\varphi}$ is the tangential stress in the middle surface. From Hooke's law we have

$$
\epsilon_{\varphi}=\frac{w}{R}=\frac{1}{E}\left(\sigma_{\varphi}-\nu \frac{n}{R t}\right),
$$

and we obtain

$$
\sigma_{\varphi}=E \frac{w}{R}+\nu \frac{n}{R t} .
$$

Finally the bending moment $m$ is given by the shell formula [ $t]$

$$
m=\frac{1}{12} \frac{E t^{3} R}{1-\nu^{2}} \frac{d^{2} w}{d x^{2}} .
$$


Eliminating $m, q$ and $\sigma_{\varphi}$ from (11), (12), (14) and (15) we obtain the differential equation ${ }^{1}$

$$
\frac{E}{12\left(1-\nu^{2}\right)} \frac{d^{2}}{d x^{2}}\left(t^{3} \frac{d^{2} w}{d x^{2}}\right)+\frac{E t}{R^{2}} w=-\left(\frac{1}{2}+\nu\right) \frac{n}{R^{2}} .
$$

The boundary conditions are here again that $w$ and its derivatives tend to zero for $x \rightarrow \infty$, whereas at the minimum cross-section

$$
x=0: \quad \frac{d w}{d x}=0, \quad q=0 .
$$

Trying again a power series solution in $t^{-1}$, we obtain the simple solution

$$
w=-\frac{2\left(1-\nu^{2}\right)(1+2 \nu)}{5-4 \nu^{2}} \frac{n}{E} \frac{1}{\delta+x^{2} / 2 R},
$$

which satisfies both the differential equation and the boundary conditions.

The bending stress at the inner surface of our shell is given by

$$
\sigma_{b}=\frac{1}{2} \frac{E}{1-\nu^{2}} t \frac{d^{2} w}{d x^{2}}=\frac{n}{\delta R} \frac{1+2 \nu}{5-4 \nu^{2}} \frac{1-\frac{3}{2} \xi^{2}}{\left[1+\left(\xi^{2} / 2\right)\right]^{2}},
$$

where $\xi$ is defined by (9). The maximum stress occurs at the inner surface in the minimum section

$$
\sigma_{\max }=\frac{n}{\delta R}\left[1+\frac{1+2 \nu}{5-4 \nu^{2}}\right] ;
$$

the nominal stress is given by $n / \delta R$, and the stress concentration factor is therefore

$$
K=\frac{(6-4 \nu)(1+\nu)}{5-4 \nu^{2}},
$$

varying from 1.2 for $\nu=0$ to 1.5 for $\nu=0.5$.

4. Alternative approach. The stress distribution depends on the two coordinates $x$ and $z$ in Fig. 2. Putting

$$
x=(R \delta)^{1 / 2} \xi, \quad z=-\delta \eta,
$$

we may rewrite the basic equations and boundary conditions (the generalized plane stress equations for the strip problem, and the axially symmetric equations for the cylindrical bar problem) in terms of the new independent variables $\xi$ and $\eta$. Developing these equations with respect to the small parameter $\epsilon=\delta / R$, and assuming a similar development for the stresses, a development for the stress concentration factor may be obtained with respect to $\epsilon$. It may well be that such a development does not converge for any finite value of $\epsilon$ but even then it will represent an asymptotic expansion. The first term has already been obtained by this method for the strip problem, with the result

$$
K=2+0(\epsilon),
$$

confirming our elementary result of Sec. 2 for $\epsilon \rightarrow 0$. Further terms in the strip problem and the similar development for the cylindrical bar problem are now being investigated.

${ }^{1}$ The author is indebted to his collaborator Mr. J. G. Lekkerkerker who pointed out an error in the original analysis. 


\section{REFERENCES}

1. R. C. J. Howland, On the stresses in the neighbourhood of a circular hole in a strip in tension, Phil. Trans. Roy. Soc., London A229, 48 (1930)

2. R. B. Heywood, Designing by photoelasticity, Chapman and Hall, London, 1952, p. 268

3. C. B. Ling, Stresses in a circular cylinder having a spherical cavity under tension, Quart. Appl. Math. 13, 381 (1956)

4. C. B. Biezeno und R. Grammel, Technische Dynamik, Springer, Berlin, 1939, p. 462

\section{THE ANISOTROPIC TENSORS*}

\section{By G. F. SMITH AND R. S. RIVLIN (Brown University)}

1. Introduction. We consider an $n$th order Cartesian tensor with components $a_{i_{1}, \ldots, \ldots i_{n}}\left(i_{p}=1,2,3\right)$ in the rectangular Cartesian coordinate system $x_{i}(i=1,2,3)$ and components $a_{i_{1},}^{*}, \ldots i_{n}$ in the rectangular Cartesian coordinate system $x_{i}^{*}$, where $x_{i}$ and $x_{i}^{*}$ are related by the orthogonal transformation

with

$$
x_{i}^{*}=s_{i j} x_{i}
$$

$$
s_{i j} s_{i k}=\delta_{j k},
$$

where $\delta_{j k}$ is the Kronecker delta.

Then,

$$
a_{i_{1} i_{3} \cdots i_{n}}^{*}=s_{i_{1} i_{2}} s_{i_{2} i_{2}} \cdots s_{i_{n} i_{n}} a_{i_{1} i_{2} \cdots i_{n}} .
$$

If

$$
a_{i_{1} i_{1}, \ldots i_{n}}^{*}=a_{i_{1} i_{2}, \ldots i_{n}},
$$

for all $s_{i j}$ satisfying (1.2), then $a_{i_{1}}, \cdots i_{n}$ are said to be the components of an isotropic tensor. If the relation (1.4) is valid only for a subgroup $\{\mathbf{T}\}$ of the group of transformations defined by (1.1) and (1.2) we shall describe $a_{i_{1} i_{3} \cdots i_{n}}$ as an anisotropic tensor. $a_{i_{1} i}, \ldots i_{n}$ may then be described as invariant under the group of transformations. The isotropic tensor is, of course, invariant under the orthogonal group.

If (1.3) and (1.4) are valid for a transformation $s_{i j}$, then it follows that

$$
a_{i_{1} i_{1}, \cdots i_{n}}=s_{i_{1} i_{2}} s_{i_{2} i_{2}} \cdots s_{i_{n} i_{n}} a_{i_{1} j_{2}, \cdots i_{n}} \text {. }
$$

If $s_{i j}$ satisfies the relation (1.2), then we readily see from (1.5) that

$$
\begin{aligned}
a_{i_{1} i_{2}} \cdots i_{n} & =s_{i_{1} i_{2}} s_{i_{2} i_{1}} \cdots s_{i_{n} i_{n}} a_{i_{1} i_{2} \cdots i_{n}} \\
& =s_{i_{1} i_{1}} s_{i_{3} i_{2}} \cdots s_{i_{n} i_{n}} a_{i_{1} i_{2}} \cdots i_{n}
\end{aligned}
$$

It is shown in Sec. 3 that any tensor which is invariant under the group of transformations $\{\mathbf{T}\}$ may be expressed as the sum of a number of terms, formed from the outer products of a finite set of tensors, with scalar $\dagger$ coefficients. This finite set of tensors,

"Received June 27, 1956. The results presented in this paper were obtained in the course of research sponsored by the Office of Ordnance Research, U. S. Army, under Contract No. DA-19-020-3487.

tThroughout this paper, we shall employ the term scalar in the following sense. If corresponding quantities $\varphi$ and $\varphi^{*}$ are defined in the coordinate system $x_{i}$ and in each coordinate system $x_{*}^{*}$, into which $x_{i}$ is transformed by the group of transformations $\{T\}$, then if $\varphi^{*}=\varphi$, we shall say that $\varphi$ is scalar with respect to the transformation group $\{T\}$ or, more briefly, scalar. 